\title{
An Extension of Some Results Due to Cox and Leland
}

\author{
Andrew P. Leung, Wen Shi \\ Monash University, Clayton, Australia \\ Email: andrew.leung@monash.edu, apleung@gmail.com,wen.shi@monash.edu
}

Received June 10, 2013; revised July 21, 2013; accepted August 9, 2013

Copyright (C) 2013 Andrew P. Leung, Wen Shi. This is an open access article distributed under the Creative Commons Attribution License, which permits unrestricted use, distribution, and reproduction in any medium, provided the original work is properly cited.

\begin{abstract}
We investigate an optimal portfolio allocation problem between a risky and a risk-free asset, as in [1]. They obtained explicit conditions for path-independence and optimality of allocation strategies when the price of the risky asset follows a geometric Brownian motion with constant asset characteristics. This paper analyzes and extends their results for dynamic investment strategies by allowing for non-constant returns and volatility. We adopt a continuous-time approach and appeal to well established results in stochastic calculus for doing so.
\end{abstract}

Keywords: Path Independence; Dynamic Asset Allocation; Dynamic Optimization; Calculus of Variations

\section{Introduction}

Beginning with [2], diffusion processes have been the standard for modeling asset returns, despite empirical evidence that returns are not normally distributed. Dynamic asset allocations based on these processes have been prominent, for example see $[3,4]$ and $[5,6]$ provide a survey of this topic to the early 1990s.

Based on the work of [7] and [1,2] derived criteria for controls to optimize an investor's objectives. They restricted the case to a portfolio with only two assets, a risky one paying no dividends and a risk-free one with the price of the risky asset following a geometric Brownian motion process.

The restriction to a single risky asset involves no significant loss of generality since the setting can be taken as a mutual fund. [8] shows that if geometric Brownian motion models are adopted, the separation theorem of mutual funds can be applied: in a portfolio problem of allocating wealth across many risky assets, the problem can be reduced to that of choosing amongst combinations of a few funds formed from these assets.

However, [1] assume constancy of asset characteristics, which is restrictive. In addition, their use of the discretetime binomial model, converging in continuous-time by limiting the time intervals, is cumbersome and detracts from the economics of the issue. Nonetheless, their result of efficiency of path-independent strategies has been extensively cited in the literature, especially in the studies for hedge funds.

[6] claim that, although the results presented by [1] were not well known at that time, path-independence of a strategy is often necessary for such a dynamic strategy to be optimal. In their study of hedge fund performance, when constructing a payoff function [9] stipulate that payoff must be a path-independent non-decreasing function of the index value, derived from [1].

[10] extend the relevance of path-independence to the case when prices of risky assets follow an exponential Lévy process. On the other hand, path-independent strategies are not always attractive. [11] show that pathdependent strategies are suboptimal for risk-averse investors when the pricing model is a function of the risky asset price at terminal time. However, and not surprisingly, path-dependent strategies are preferred if the pricing model of the risky assets is itself path- dependent.

In this paper, we extend the results of [1] for more general asset return processes. We assume that the price of the riskless asset grows deterministically at a variable interest rate, and the price for the risky asset follows geometric Brownian motion, with both the drift and volatility being variable over both time and the stock price. Such a model mitigates some of the difficulties in explaining long-observed features of the implied volatility surface for option pricing. Hence it is possible to model derivatives more realistically.

Detailed references for such stochastic processes may be found in $[12,13]$. Without loss of generality, we consider a world with a risky asset and a riskless asset, as in [1]. We establish our results by application of a continuous-time approach and the use of partial differential 
equations (PDEs), rather than through stochastic calculus. We obtain explicit results for general dynamic strategies which allow for uncertainty as modeled in diffusion processes. These results extend those of [1].

Our results are concerned with maximizing some form of investor utility. In most former studies, when dealing with utility maximization, a particular form of utility function is specified. For example, a HARA utility is considered in [2]; an iso-elastic utility in [14]; and a CRRA power utility in [15]. While the Hamilton-JacobiBellman equation is a popular tool for utility maximization problems, [16] criticizes the use of an arbitrary "bequest function" as the boundary condition in [2]; the boundary behavior around zero terminal wealth may be inconsistent with his "bequest function". In our approach, the boundary condition is taken as an arbitrary utility function of terminal wealth, thereby avoiding this problem. [17] gives a more detailed review of expected utility maximization for strategies involving a risky and a riskless asset. Although he does not approach this problem in full generality, using the example of a power utility function, he shows how other cases can be solved with little effort.

In the working papers by $[18,19]$, for a given utility function, the Feynman-Kac formula is used to find controls satisfying certain PDEs for utility maximization. We show that the Feynman-Kac formula can generally provide the solution to a control in terms of its terminal value. We also show that the terminal value satisfies some concave utility, without specifying its functional form.

The paper is organized as follows. First, for simplicity, we assume no cash flows, which corresponds to the pure "bequest" case of [7]. This assumption is then later relaxed.

- Section 2 extends Proposition 1 of [1] for necessary and sufficient conditions for an investment strategy to be feasible, where the controls of the strategy are given as functions of time and the value of the risky asset.

- Section 3 develops necessary and sufficient conditions for an investment strategy to be path-independent, with controls defined as functions of time and the value of the portfolio (wealth). These results extend Proposition 2 of [1].

- Section 4 establishes necessary and sufficient conditions for an investment strategy to optimize a concave utility, while imposing no constraints on portfolio allocations. This extends Proposition 3 of [1].

- Sections 5 and 6 consider the case of non-negative allocations.

- Section 7 considers the situation when cash with drawals are admissible.

- Section 8 concludes.

\section{Controls Based on Stock Price $s$}

Suppose $W(s . t)=G+H$ is total wealth, invested in a risky asset $G$ and a riskless asset $H$. Suppose also that

$$
\frac{\mathrm{d} s}{\mathrm{~s}}=\mu \mathrm{d} t+\sigma \mathrm{d} B
$$

is the process for the risky asset, where $B(t)$ is Brownian motion ${ }^{1}$, and both $\mu, \sigma$ may depend on both $t$ and $s$. Itô's theorem provides that:

$$
\mathrm{d} W=W_{t} \mathrm{~d} t+W_{s} \mathrm{~d} s+\frac{1}{2} W_{s s}(\mathrm{~d} s)^{2} .
$$

Let $r(t)$ denote the riskless rate at time $t$. This is generally independent of the stock price $s$, by virtue of being riskless.

Then, when there are no cash withdrawals or injections (i.e. the strategy is self-financing),

$$
\mathrm{d} G+\mathrm{d} H=\frac{\mathrm{d} s}{s} G+r H \mathrm{~d} t,
$$

so that

$$
\begin{aligned}
& \mathrm{d} W=\mu G \mathrm{~d} t+r H \mathrm{~d} t+\sigma G \mathrm{~d} B \\
& =W_{t} \mathrm{~d} t+s W_{s}(\mu \mathrm{d} t+\sigma \mathrm{d} B)+\frac{1}{2} \sigma^{2} s^{2} W_{s s} \mathrm{~d} t .
\end{aligned}
$$

This implies that

$$
\mu G+r H=r W+(\mu-r) G=W_{t}+\mu s W_{s}+\frac{1}{2} \sigma^{2} s^{2} W_{s s}
$$

and

$$
G=s W_{s} .
$$

thus

$$
W_{t}+\mu s W_{s}+\frac{1}{2} \sigma^{2} s^{2} W_{s s}-r W=(\mu-r) s W_{s}
$$

and so

$$
W_{t}+r s W_{s}+\frac{1}{2} \sigma^{2} s^{2} W_{s s}-r W=0 .
$$

These equalities are consistent with the conditions of Proposition 1 of [1]. Note that the expected return on the risky asset $\mu$ does not appear in 5 .

Differentiating Equation (5) with respect to $s$ yields:

$$
\begin{aligned}
& W_{s t}+r s W_{s s}+r_{s} s W_{s}+r W_{s}+\frac{1}{2} \sigma^{2} s^{2} W_{s s s} \\
& +\sigma^{2} s W_{s s}+\sigma \sigma_{s} s^{2} W_{s s}=r W_{s}+r_{s} W .
\end{aligned}
$$

Multiplying this last equality by $s$, we get

$$
\begin{aligned}
& s W_{s t}+r s^{2} W_{s s}+r_{s} s^{2} W_{s}+r s W_{s}+\frac{1}{2} \sigma^{2} s^{3} W_{s s s} \\
& +\sigma^{2} s^{2} W_{s s}+\sigma \sigma_{s} s^{3} W_{s s}=r s W_{s}+r_{s} s W .
\end{aligned}
$$

${ }^{1}$ We use $B(t)$ rather than the more conventional $B_{t}$, as subscripts in this paper are reserved solely for derivatives. 
On the other hand since $G=s W_{s}$, we have

$$
\begin{gathered}
G_{t}=s W_{s t} \\
G_{s}=W_{s}+s W_{s s} \\
G_{s s}=2 W_{s s}+s W_{s s s} .
\end{gathered}
$$

Hence:

$$
\begin{aligned}
& G_{t}+r s G_{s}+r_{s} s^{2} W_{s}+\frac{1}{2} \sigma^{2} s^{2} G_{s s} \\
& +\sigma \sigma_{s} s\left(s G_{s}-G\right)=r G+r_{s} s W
\end{aligned}
$$

and, as $r_{s}=0$, this may be formalized as:

Proposition 1: The controls $W(s . t)$ and $G(s, t)$ satisfy the PDEs:

$$
\begin{gathered}
W_{t}+r s W_{s}+\frac{1}{2} \sigma^{2} s^{2} W_{s s}-r W=0 \\
G_{t}+s\left(r+\sigma \sigma_{s}\right) G_{s}+\frac{1}{2} \sigma^{2} s^{2} G_{s s}-\left(r+s \sigma \sigma_{s}\right) G=0 .
\end{gathered}
$$

Remark: Thus proposition 1 of [1] will not apply for $G$ if $\sigma_{s} \neq 0$. However note that $G$ has the same form as $W$, but with $r$ replaced by $r+\sigma \sigma_{s}$.

\section{Controls Based on Wealth W}

Since $W=W(s, t)$ we now consider $G$ and $H$ as functions of $(W, t)$. This corresponds to Proposition 2 of [1].

On this basis

$$
\begin{gathered}
G_{t \mid s}=G_{t \mid W}+G_{W} W_{t} \\
G_{s}=G_{W} W_{s} \\
G_{s s}=G_{W} W_{s s}+G_{W W} W_{s}^{2}
\end{gathered}
$$

Hence the left hand side of Equation (7) becomes

$$
\begin{aligned}
G_{t} & +r s G_{s}+\frac{1}{2} \sigma^{2} s^{2} G_{s s}-r G \\
= & G_{t \mid W}+G_{W} W_{t}+r s G_{W} W_{s} \\
& +\frac{1}{2} \sigma^{2} s^{2}\left(G_{W} W_{s s}+G_{W W} W_{s}\right)-r G \\
= & G_{t \mid W}+G_{W}\left(W_{t}+r s W_{s}+\frac{1}{2} \sigma^{2} s^{2} W_{s s}\right) \\
& +\frac{1}{2} \sigma^{2} s^{2} G_{W W} W_{s}-r G \\
= & G_{t \mid W}+r W G_{W}+\frac{1}{2} \sigma^{2} s^{2} G_{W W} W_{s}^{2}-r G \\
= & G_{t \mid W}+r W G_{W}+\frac{1}{2} \sigma^{2} G^{2} G_{W W}-r G .
\end{aligned}
$$

For the right hand side of Equation (7):

$$
\sigma_{s}=\sigma_{W} W_{s}
$$

and so

$$
\begin{aligned}
s \sigma \sigma_{s}\left(G-s G_{s}\right) & =s \sigma \sigma_{W} W_{s}\left(G-s G_{W} W_{s}\right) \\
& =\sigma \sigma_{W} G^{2}-s^{2} \sigma \sigma_{W} G_{W} W_{s}^{2}
\end{aligned}
$$

Hence we have:

Proposition 2: The control $G(W, t)=s W_{s}$, viewed as a function of wealth, satisfies:

$$
G_{t \mid W}+r W G_{W}+\frac{1}{2} \sigma^{2} G^{2} G_{W W}-r G=\sigma \sigma_{W} G^{2}\left(1-G_{W}\right)
$$

Remark: This is the same as proposition 2 of [1] when $\sigma_{W}=0$.

\section{Controls That Are Compatible with a Concave Utility}

Consider a control $G(s, t)$ that maximizes an expected utility of terminal wealth $U$ at time $T$ :

$$
U=\mathbf{E}_{P}[u(W)]
$$

for some utility function $u(W)$ and where $P$ is the physical measure under the process in 1.

Then the Equation (5) is, regarded as a parabolic partial differential equation:

$$
W_{t}+p W_{s}+\frac{1}{2} q W_{s s}-r W=0
$$

where $r=r(t)$ is a function of $t$ and $p=r s$ and $q=s^{2} \sigma^{2}$ are functions of $(s, t)$.

The solution is given by the Feynman-Kac formula. The solution, expressed as a stochastic expectation, is:

$$
\begin{aligned}
W(s, t) & =\mathbf{E}_{Q}[\psi(S) v(t) \mid s(t)=s] \\
& =v(t) \mathbf{E}_{Q}[\psi(S)] .
\end{aligned}
$$

Here $s=s(T)$ and $\psi(S)=W(S, T)$ for a given value of $T$, and

$$
v(t)=\exp -\int_{t}^{T} r(z) \mathrm{d} z .
$$

The expectation $\mathbf{E}_{Q}$ is taken with respect to the risk neutral process:

$$
\mathrm{d} s=r s \mathrm{~d} t+\sigma s \mathrm{~d} B .
$$

Remark: It is known there are various conditions for the Feynman-Kac formula to hold, which are set out in the Appendix. A condition that $q$ be bounded above zero is not onerous, as we are dealing with a risky asset. Some of these conditions may be relaxed significantly, and will be discussed in a further paper.

The probability density $\pi(s, t ; S, T)$ of $s$ at time $t$ is governed by the Kolmogorov backward Equation

$$
\pi_{t}=-p \pi_{s}-\frac{1}{2} q \pi_{s s}
$$

and also the Kolmogorov forward equation 


$$
\pi_{T}=-(p \pi)_{S}+\frac{1}{2}(q \pi)_{S S} .
$$

The conditions for these results are also set out in the Appendix.

The critical implication of 9 is that $W(s, t)$, and therefore $G(s, t)=s W_{s}$, is completely determined by the terminal wealth $\psi(S)$, along with an initial condition, say $W\left(s_{0}, 0\right)=1$ for some initial stock price $S_{0}$.

In addition, since $G(s, t)=s W_{s}$ satisfies the similar PDE in 7, we have:

$$
G(s, t)=\mathbf{E}_{R}\left[S \psi_{S}(S) w(t) \mid s(t)=s\right],
$$

where the expectation $\mathbf{E}_{R}$ is taken with respect to the process:

$$
\mathrm{d} s=\left(r+s \sigma \sigma_{s}\right) s \mathrm{~d} t+\sigma s \mathrm{~d} B
$$

and

$$
w(t)=\exp -\int_{t}^{T}\left(r+s \sigma \sigma_{s}\right) \mathrm{d} z
$$

This implies that $G(s, t) \geq 0$ if $\psi_{S}(S) \geq 0$.

\section{Optimization}

Thus for the utility function $u(W)$, it suffices to find $\psi(S)$ so as to maximize:

$$
\begin{aligned}
U & =\mathbf{E}_{P}[u(W)] \\
& =\int \bar{\pi}\left(s_{0}, 0 ; S, T\right) u[\psi(S)] \mathrm{d} S .
\end{aligned}
$$

Here the expectation $\mathbf{E}_{P}$ and density $\bar{\pi}$ relate to the physical stock process:

$$
\mathrm{d} s=\mu \mathrm{s} t+s \sigma \mathrm{d} B
$$

rather than to 10 .

This is subject to the initial condition:

$$
\begin{aligned}
W(s, 0) & =v(0) E_{Q}[\psi(S)] \\
& =v(0) \int \pi\left(s_{0}, 0 ; S, T\right) \psi(S) \mathrm{d} S .
\end{aligned}
$$

where the expectation $\mathbf{E}_{Q}$ is subject to the risk neutral process in 10.

The Lagrangian is

$$
\mathcal{L}=\int[\bar{\pi} u[\psi(S)]-\lambda \pi \psi(S)] \mathrm{d} S .
$$

Let $\Delta \psi$ be a variation in $\psi$. The resulting variation in $\mathcal{L}$ is, to the second order:

$$
\Delta \mathcal{L}=\int \Delta \psi\left[\bar{\pi} u_{W}+\frac{1}{2} \bar{\pi} u_{W W} \Delta \psi-\lambda \pi\right] \mathrm{d} S
$$

Since $\Delta \mathcal{L} \leq 0$ for any variations $\Delta \psi$, the first order condition is

$$
\bar{\pi} u_{W}[\psi(S)]=\lambda \pi
$$

where $\lambda>0$. Thus given $u(W)$, the function $\psi(S)$ may be found from

$$
u_{W}[\psi(S)]=\lambda \frac{\pi\left(s_{0}, 0 ; S, T\right)}{\bar{\pi}\left(s_{0}, 0 ; S, T\right)}
$$

with $\lambda$ being chosen to satisfy the initial condition 13 .

The second order condition is $u_{w w} \leq 0$, so that a concave utility is required. The general solution for $W(s, t)$ in terms of $\psi(S)$ is then given by 9 . In the general case with $r, \mu, \sigma$ not constant, we thus have the following extension of the existential results of Proposition 3 of [1]:

Proposition 3 A path independent strategy $W(s, t)$ can be found to optimize a given concave utility $u(W)$ if, and only if, a solution $\psi(S)$ can be found to satisfy:

$$
u_{W}[\psi(S)]=\lambda \frac{\pi\left(s_{0}, 0 ; S, T\right)}{\bar{\pi}\left(s_{0}, 0 ; S, T\right)} .
$$

Remark: This is without qualification as to the existence of a solution to 8 . In the case that $u(W)$ is given, the Inada conditions provide that $u_{W}$ is invertible on $(0, \infty)$, so that

$$
\psi(S)=u_{W}^{-1}\left[\lambda \frac{\pi\left(s_{0}, 0 ; S, T\right)}{\bar{\pi}\left(s_{0}, 0 ; S, T\right)}\right] .
$$

In the case that $\psi(S)$ is given, the condition $\psi_{S} \geq 0$ is sufficient to determine $u_{W}$. However none of these conditions is mentioned in Proposition 3 of [1].

Example: [1] assume constant returns and volatilities $r, \mu, \sigma$. In this case, it is well known that $\ln S / s_{0}$ is normally distributed, with mean

$$
\mu T-\frac{1}{2} \sigma^{2} T
$$

(physical measure) or

$$
r T-\frac{1}{2} \sigma^{2} T
$$

(risk neutral measure), and variance $\sigma^{2} T$ at time $T$ :

$$
\bar{\pi}\left(s_{0}, 0 ; S, T\right)=\frac{1}{\sqrt{2 \pi T} \sigma S} \exp -\left[\frac{\left(\ln \frac{S}{s_{0}}-\mu T+\frac{1}{2} \sigma^{2} T\right)^{2}}{2 \sigma^{2} T}\right]
$$

while

$$
\pi\left(s_{0}, 0 ; S, T\right)=\frac{1}{\sqrt{2 \pi T} \sigma S} \exp -\left[\frac{\left(\ln \frac{S}{s_{0}}-r T+\frac{1}{2} \sigma^{2} T\right)^{2}}{2 \sigma^{2} T}\right] .
$$


Hence 14 becomes:

$$
\begin{aligned}
& u_{W}[\psi(S)]=\lambda \exp \\
& -\left[\frac{\left(r^{2}-\mu^{2}\right) T^{2}-2\left(\ln \frac{S}{s_{0}}+\frac{1}{2} \sigma^{2} T\right)(r-\mu) T}{2 \sigma^{2} T}\right] \\
& =\lambda \exp \left[\frac{\left(\mu^{2}-r^{2}\right) T}{2 \sigma^{2}}\right] \cdot\left[\frac{S}{S_{0}}\right]^{\frac{r-\mu}{\sigma^{2}}} \cdot \mathrm{e}^{\frac{1}{2}(r-\mu) T} .
\end{aligned}
$$

Differentiating with respect to $S$, we also have

$$
\begin{aligned}
& u_{W W} \cdot \psi_{S}(S)=\lambda \frac{r-\mu}{\sigma^{2}} \\
& \exp \left[\frac{\left(\mu^{2}-r^{2}\right) T}{2 \sigma^{2}}\right] \cdot \frac{1}{s_{0}}\left[\frac{S}{s_{0}}\right]^{\frac{r-\mu}{\sigma^{2}-1}} \cdot \mathrm{e}^{\frac{1}{2}(r-\mu) T} .
\end{aligned}
$$

Thus $\psi_{S} \geq 0$ when $r \leq \mu$, and vice versa.

By virtue of 12 , depending on whether $r \leq \mu$, we have a proof of Proposition 3 of [1].

\section{Extension of Utility Characterization}

It is of interest to consider whether the allocation to the risky asset $G(s, t)$ is non-negative under more general conditions than indicated in Proposition 3 of [1].

Proposition 4 Suppose $\mu, \sigma^{2}$ are non-stochastic (i.e. independent of $s$ ) and $r(t) \leq \mu(t)$. Then a strategy $W(s, t)$ can be found to optimize a concave utility $u(W)$ with $G(s, t) \geq 0$.

Proof. Given $u(W)$, the strategy $W(s, t)$ is given by 14 and the Kac-Feynman formula 9 . We also note the relation 12 , which shows that $G(s, t) \geq 0$. if $\psi_{S}(S) \geq 0$ at time $T$.

Differentiating 14 with respect to $S$, we have:

$$
u_{W W} \cdot \psi_{S}=\lambda \frac{\pi_{S} \bar{\pi}-\pi \bar{\pi}_{S}}{\bar{\pi}^{2}} .
$$

Since $u_{W W} \leq 0, \quad$ it suffices to show that $(\ln \pi)_{S} \leq(\ln \bar{\pi})_{S}$. The variant of Girsanov's theorem, as proved in the Appendix, confirms this result.

Remark: The conditions are sufficient, but by no means necessary. The Appendix shows that the density $\rho(Z, T)$ of the risk neutral process for $z=\ln s$ :

$$
\mathrm{d} z=\left(\mu-\frac{1}{2} \sigma^{2}\right) \mathrm{d} t+\sigma \mathrm{d} B .
$$

with stochastic $\sigma$, is central to this issue. In particular, if the risk premium $\mu-r$ is non-stochastic, and $\ln \rho(Z, T)$ is concave in $Z$, then the result also holds. This situation may be investigated by noting that $\rho(Z, T)$ satisfies a parabolic PDE, which can in turn be investigated by the eigenfunctions of the operator

$$
L: \pi \rightarrow\left[\left(\mu-\frac{1}{2} \sigma^{2}\right) \pi\right]_{z}+\frac{1}{2}\left(\sigma^{2} \pi\right)_{z z} .
$$

\section{Constrained Strategies}

The above discussion does not constrain the allocations to both the risky asset and the riskless asset to be nonnegative, which is often a requirement in practice. For this to apply, we have the additional constraints on terminal wealth:

$$
0 \leq S \psi_{S}(S) \leq \psi(S) .
$$

If $\mu \geq r$, then Proposition 4 provides conditions for $G(s, t) \geq 0$. To provide that $G(s, t) \leq W(s, t)$, we need to have the terminal condition:

$$
S \psi_{S}(S) \leq \psi(S) .
$$

If this holds, and $\sigma_{s} \geq 0$, then Equation (12) implies:

$$
\begin{aligned}
& G(s, t)=\mathbf{E}\left[S \psi_{S}(S) w(t) \mid s(t)=s\right] \\
& \leq \mathbf{E}\left[\psi_{S}(S) v(t) \mid s(t)=s\right]=W(s, t) .
\end{aligned}
$$

To ensure that 15 holds, consider the Lagrangian:

$$
\begin{aligned}
& \mathcal{L}=\int\{\bar{\pi} u[\psi(S)]-\lambda \pi \psi(S) \\
& \left.-\kappa(S)\left[\psi(S)-S \psi_{S}(S)\right]\right\} \mathrm{d} S .
\end{aligned}
$$

Let $\Delta \psi$ be a variation in $\psi$ such that $S \Delta \psi \rightarrow 0$ when $S \rightarrow \infty$. The variation in $\mathcal{L}$ is, to the second order:

$$
\begin{aligned}
\Delta \mathcal{L} & =\int \Delta \psi\left[\bar{\pi} u_{W}+\frac{1}{2} \bar{\pi} u_{W W}(\Delta \psi)^{2}-\lambda \pi \Delta \psi-\kappa\right] \mathrm{d} S \\
& +\int \kappa S \Delta \psi_{S} \mathrm{~d} S \\
& =\int \Delta \psi\left[\bar{\pi} u_{W}+\frac{1}{2} \bar{\pi} u_{W W} \psi(\Delta \psi)^{2}-\lambda \pi-\kappa\right] \mathrm{d} S \\
& -\int \Delta \psi\left(\kappa+\kappa_{S} S\right) \mathrm{d} S
\end{aligned}
$$

The first order condition is thus:

$$
\bar{\pi} \cdot u_{W}[\psi(S)]=\lambda \pi+2 \kappa+\kappa_{S} S
$$

which can be written:

$$
\left(S^{2} \kappa\right)_{S}=S\left[\bar{\pi} \cdot u_{W}[\psi]-\lambda \pi\right],
$$

and thus integrating over $S$ :

$$
\kappa=\frac{1}{S^{2}} \int_{0}^{S}\left[\bar{\pi} \cdot u_{W}[\psi(z)]-\lambda \pi(z)\right] \mathrm{d} z \geq 0 .
$$

The second order condition is as before:

$$
\bar{\pi} \cdot u_{W W}[\psi(S)] \leq 0 .
$$

This leads to the following result:

Proposition 5: Given a concave utility $u(W)$, and 
$\sigma_{s} \geq 0$, the strategy $W(s, t)$, given by the KacFeynman formula 9, provides optimality over nonnegative allocations to the riskless asset, only if there is a solution $\psi(S)$ of:

$$
\int_{0}^{S}\left[\bar{\pi} \cdot u_{W}[\psi(z)]-\lambda \pi(z)\right] \mathrm{d} z \geq 0
$$

for some $\lambda \geq 0$.

Remark: These are weaker conditions than provided in Proposition 3, as we are seeking optimality over a smaller class of allocations. Even weaker conditions may be found if the class of allocations is restricted to where both the risky and riskless assets are constrained to be non-negative.

\section{Allowance for Cash Flows}

The previous relations can be extended to accommodate portfolios with cash withdrawals. Let us now consider the situation when an investor is allowed to withdraw from their investment, at a rate $K \geq 0$. Such as before, we discuss the cash withdrawn from the portfolio in two cases, a function of price of the risky asset and time, $K(s, t)$, or a function of total wealth and time, $K(W, t)$.

Total wealth $W$ then obeys the generalised relation:

$$
\mathrm{d} W=\mathrm{d} G+\mathrm{d} H=\frac{\mathrm{d} s}{\mathrm{~s}} G+r H \mathrm{~d} t-K \mathrm{~d} t .
$$

\subsection{Controls That Are Functions of the Value of the Risky Asset and Time}

In analogy with section 2 consider the case where the controls, $G, H$ and $K$, are all functions of $(s, t)$, where the process of $s$ is the same as in 1 .

Allowing for cash withdrawals, 2 generalizes to

$$
\begin{aligned}
\mathrm{d} W & =\mu G \mathrm{~d} t+r H \mathrm{~d} t+\sigma G \mathrm{~d} B-K \mathrm{~d} t \\
& =W_{t} \mathrm{~d} t+s W_{s}(\mu \mathrm{d} t+\sigma \mathrm{d} B)+\frac{1}{2} \sigma^{2} s^{2} W_{s s} \mathrm{~d} t .
\end{aligned}
$$

This implies that

$$
r W+(\mu-r) G-K=W_{t}+\mu s W_{s}+\frac{1}{2} \sigma^{2} s^{2} W_{s s}
$$

and the same condition as in 4 , which is consistent with Proposition 1 of [1] that

$$
G=s W_{s}=s\left(G_{s}+H_{s}\right)
$$

and hence 5 generalizes to:

$$
W_{t}+r s W_{s}+\frac{1}{2} \sigma^{2} s^{2} W_{s s}-r W+K=0 .
$$

It may be shown similarly that 7 generalizes to:

$$
\frac{1}{2} \sigma^{2} s^{2} G_{s s}+r s G_{s}+G_{t}-r G+s K_{s}=\sigma \sigma_{s} s\left(G-s G_{s}\right) .
$$

Now we can formalize the above results as:

Proposition 6: Necessary and sufficient conditions for the differentiable functions $G(s, t), W(s, t)$ and $K(s, t)$ to be the controls of a self-financing investment strategy are that:

$$
\begin{gathered}
W_{t}+r s W_{s}+\frac{1}{2} \sigma^{2} s^{2} W_{s s}-r W+K=0 \\
G_{t}+s\left(r+\sigma \sigma_{s}\right) G_{s}+\frac{1}{2} \sigma^{2} s^{2} G_{s s}-\left(r+s \sigma \sigma_{s}\right) G+s K_{s}=0 \\
G=s W_{s}
\end{gathered}
$$

for all $s$ and $t$.

Notice that Proposition 1 of [1] is a special case of this generalized form with a constant diffusion for price of the risky asset, that is $\sigma_{s}=0$.

\subsection{Controls That Are Functions of the Value of the Portfolio Wealth and Time}

In analogy with section 3, consider the situation when the controls, $G$ and $K$, are functions of $(W, t)$.

As $K$ is a control, we also have:

$$
K_{s}=K_{W} W_{s} \text {. }
$$

Then Equation (7) can be shown to generalize to:

Proposition 7: The control $G(W, t)=s W_{s}$, viewed as a function of wealth, satisfies:

$$
\begin{aligned}
& G_{t \mid W}+(r W-K) G_{W}+\frac{1}{2} \sigma^{2} G^{2} G_{W W} \\
& -\left(r-K_{W}\right) G=\sigma \sigma_{W} G^{2}\left(1-G_{W}\right) .
\end{aligned}
$$

\subsection{Compatibility with Investor Objectives}

We now consider if the processes of controls $G$ and $K$ are compatible with rational investor objectives.

Express 18 to get

$$
W_{t}+r s W_{s}+\frac{1}{2} \sigma^{2} s^{2} W_{s s}=(r-k) W,
$$

where, without loss of generality, we write

$$
K=k(s, t) W \text {. }
$$

This is again a parabolic PDE in $W$, and the FeynmanKac formula can be applied to find a solution as:

$$
W(s, t)=v(t) \mathbf{E}_{Q}[\psi(S) \gamma(t) \mid s(t)=s],
$$

but now the discount factor includes:

$$
\gamma(s, t)=\exp \left[\int_{t}^{T} k(s, \tau) \mathrm{d} \tau\right] .
$$

As before, the wealth $W$ is completely determined by the terminal wealth $\psi(S)$, along with the control $k(s, t)$.

In the case with cash withdrawals are admissible, we 
consider not only the utility from terminal wealth for an investor, but also the utility from consumption financed by the cash withdrawals $K=k(s, t) W$. Therefore, the problem of choosing optimal portfolio and consumption rules for an investor over a period of $T$ is to maximize an aggregate utility of the following form:

$$
U=\mathbf{E}_{P}\left\{u[\psi(S)]+\int_{0}^{T} c(K) \mathrm{d} \tau\right\} .
$$

The function $c(K)$ is the utility from consumption, with $c_{K} \geq 0$. The initial and terminal times are specified at $t=0$ and $t=T$, as is the initial condition that some initial stock price $s(0)=s_{0}$. The expectation $\mathbf{E}_{P}$ is specified as before in Section 4 for the physical stock process.

The Lagrangian is then given by:

$$
\begin{aligned}
& \mathcal{L}=\mathbf{E}_{P}\left[u[\psi(S)]+\int_{0}^{T} c(K) \mathrm{d} \tau\right] \\
& -\lambda \nu(0) \mathbf{E}_{Q}[\psi(S) \gamma(t) \mid s(0)=s] .
\end{aligned}
$$

This optimization problem is exactly of continuous stochastic control [20, VII.10]. Define an optimal expected value function given the stock price $s$ at time $t$ :

$$
\bar{U}(s, t)=\max _{k} \mathbf{E}_{P}\left\{u[\psi(S)]+\int_{t}^{T} c(K) \mathrm{d} \tau\right\} .
$$

The process terminates at time $T$, at which time the utility of terminal wealth is assessed, with the boundary condition

$$
\bar{U}(S, T)=u[\psi(S)] .
$$

The fundamental PDE for the control $k(s, t)$ is:

$$
0=\max _{k}\left[c+\bar{U}_{s} \mu s+\bar{U}_{t}+\frac{1}{2} \bar{U}_{s s} \sigma^{2} s^{2}\right]
$$

However, we follow an alternative, but simpler, approach. Given $\psi(S)$, consider a small variation in $k$, say $\Delta k$, localized at time $\tau$ and in state $s$ :

$$
\Delta k=\eta(s, \tau) \text { in }[\tau, \tau+\mathrm{d} \tau]
$$

for some constant $\eta$, which induces a variation $\Delta K=\eta W$. This further induces variations in the terms

$$
\int_{0}^{T} c(K) \mathrm{d} \tau
$$

and $\gamma(t)$, to the first order in $\mathrm{d} \tau$ :

$$
\begin{gathered}
\Delta c(K)=c_{K}(K) \Delta K+\frac{1}{2} c_{K K}(\Delta K)^{2} \\
\Delta \int_{0}^{T} c(K) \mathrm{d} \tau=c_{K} W \eta \mathrm{d} \tau+\frac{1}{2} c_{K K}(\eta W)^{2} \mathrm{~d} \tau \\
\Delta \gamma(s, t)=\exp \left[\int_{t}^{T} k(s, \tau) \mathrm{d} \tau\right] \cdot\left(\mathrm{e}^{\eta d \tau}-1\right)=\gamma(s, t)(\eta d \tau) .
\end{gathered}
$$

And thus:

$$
\begin{aligned}
\Delta \mathcal{L}= & \mathbf{E}_{P}\left[c_{K} \eta W \mathrm{~d} \tau+\frac{1}{2} c_{K K}(\eta W)^{2} \mathrm{~d} \tau\right] \\
& -\lambda(\eta \mathrm{d} \tau) \nu(0) \mathbf{E}_{Q}[\psi(S) \gamma(t)] .
\end{aligned}
$$

Since $\eta$ is localized at $s$, and $\mathbf{E}_{Q}[\psi(S) \gamma(t)]$ is constant, the first order condition in $\eta$ is:

$$
\bar{\pi}\left(s_{0}, 0 ; s, \tau\right) c_{K} W=\kappa
$$

for some constant $\kappa>0$.

The second order condition in $\eta$ is:

$$
c_{K K} \leq 0 \text {. }
$$

Hence we have the following result.

Proposition 8 Given concave utility functions for terminal wealth $u(W)$ and for consumption $c(K)$, and a terminal wealth with density $\psi(S)$, the optimal cash flow control is given by $K(s, t)$ satisfying:

$$
c_{K}=\frac{\kappa}{\bar{\pi}\left(s_{0}, 0 ; s, t\right) W}
$$

for some constant $\kappa>0$.

Remark: As $c_{K}$ is a decreasing function in $K$, this implies that cash withdrawals should increase in the wealth $W$ achieved, but should decrease where such wealth is less likely to be achieved. This corresponds to the conditions contained in Proposition 4 of [1].

\section{Conclusions}

In this paper, we address two related issues, based on the work by [1].

First, we examine the characteristics of optimal portfolio controls. Rather than assuming constant expected returns and volatility, we consider the more realistic situation with the expected return and volatility of risky assets are non-constant, or even stochastic.

Second, we consider whether a given investment strategy is consistent with expected utility maximization. We apply several techniques of the calculus of variations to show that, under mild conditions, optimal portfolio controls are compatible with some concave utility function. Unlike most papers in the literature, we do not specify a particular form of utility function.

It would be interesting to extend these results to more general asset models, for example where the risky asset follows a jump diffusion process, or where volatility of the return on the risky asset is itself a stochastic process.

\section{REFERENCES}

[1] J. C. Cox and H. E. Leland, "On Dynamic Investment Strategies," Journal of Economic Dynamics and Control, Vol. 24, No. 11-12, 2000, pp. 1859-1880. http://dx.doi.org/10.1016/S0165-1889(99)00095-0 
[2] R. C. Merton, "Optimum Consumption and Portfolio Rules in a Continuoustime Model," Journal of Economic Theory, Vol. 3, No. 4, 1971, pp. 373-413. http://dx.doi.org/10.1016/0022-0531(71)90038-X

[3] F. Black and M. Scholes, "The Pricing of Options and Corporate Liabilities," The Journal of Political Economy, Vol. 81, No. 3, 1973, pp. 637-654. http://dx.doi.org/10.1086/260062

[4] J. C. Cox and S. A. Ross, "The Valuation of Options for Alternative Stochastic Processes," Journal of Financial Economics, Vol. 3, No. 1-2, 1976, pp. 145-166. http://dx.doi.org/10.1016/0304-405X(76)90023-4

[5] P. Carr, H. Geman and D. Madan, "The Fine Structure of Asset Returns: An Empirical Investigation," Journal of Business, Vol. 75, No. 2, 2002, pp. 305-332. http://dx.doi.org/10.1086/338705

[6] S. Hodges and R. Clarkson, "Dynamic Asset Allocation: Insights from Theory," Philosophical Transactions: Physical Sciences and Engineering, Vol. 347, No. 1684, 1994, pp. 587-598.

[7] R. C. Merton, "Lifetime Consumption under Uncertaintiy: The Continuous Time Case," The Review of Economics and Statistics, Vol. 51, No. 3, 1969, pp. 247-257. http://dx.doi.org/10.2307/1926560

[8] S. A. Ross, "Mutual Fund Separation in Financial Theory-The Separating Distributions," Journal of Economic Theory, Vol. 17, No. 2, 1978, pp. 254-286. http://dx.doi.org/10.1016/0022-0531(78)90073-X

[9] G. S. Amin and H. M. Kat, "Hedge Fund Performance 1990-2000: Do the 'Money Machines' Really Add Value?" Journal of Financial and Quantitative Analysis, Vol. 38, No. 2, 2003, pp. 251-274. http://dx.doi.org/10.2307/4126750

[10] S. Vanduffel, A. Chernih, M. Maj and W. Schoutens, "A Note on the Suboptimality of Path-Dependent Pay-Offs in
Lévy Markets," Applied Mathematical Finance, Vol. 16, No. 4, 2009, pp. 315-330.

http://dx.doi.org/10.1080/13504860802639360

[11] S. Kassberger and T. Liebmann, "When Are Path-Dependent Payoffs Suboptimal?” 2009.

[12] F. C. Klebaner, "Introduction to Stochastic Calculus with Applications," Imperial College Press, London, 2005. http://dx.doi.org/10.1142/p386

[13] O. C. Ibe, "Markov Processes for Stochastic Modeling," Elsevier Academic Press, Waltham, 2009.

[14] M. J. Brennana, E. S. Schwartz and R. Lagnado, "Strategic Asset Allocation," Journal of Economic Dynamics and Control, Vol. 21, No. 8-9, 1997, pp. 1377-1403. http://dx.doi.org/10.1016/S0165-1889(97)00031-6

[15] J. Cvitanic and V. Polimenis, "Optimal Portfolio Allocation with Higher Mo Moments," Annals of Finance, 2008.

[16] S. P. Sethi, "A note on Mertons Optimum Consumption and Portfolio Rules in a continuous-Time Model," Journal of Economic Theory, Vol. 46, No. 2, 1988, pp. 395401. http://dx.doi.org/10.1016/0022-0531(88)90138-X

[17] A. Meucci, "Review of Dynamic Allocation Strategies: Utility Maximization, Option Replication, Insurance, Drawdown Control, Convex-Concave Management,” 2010.

[18] K. Tanaka, "Dynamic Asset Allocation under Stochastic Interest Rate and Market Price of Risk," 2009.

[19] C. Chiarella, C. Hsaio and W. Semmler, "Intertemporal Investment Strategies under Inflation Risk," University of Technology, Sydney, 2007.

[20] S. E. Dreyfus, "Dynamic Programming and the Calculus of Variations," Academic Press, Norwell, 1965.

[21] S. Janson and J. Tysk, "Feynman-Kac Formulas for BlackScholes Type Operators," Bulletin London Mathematical Society, Vol. 38, No. 2, 2006, pp. 269-282. http://dx.doi.org/10.1112/S0024609306018194 


\section{Appendix: Conditions for Known Results}

Let a general stochastic process be defined by

$$
\mathrm{d} s=p \mathrm{~d} t+\sqrt{q} \mathrm{~d} B
$$

where $p$ and $q$ are functions of $(s, t)$. Let $\pi(s, t ; S, T)$ denote the density of $S$ at time $T$, given an initial value of $(s, t)$. Derivatives $\pi_{s}, \pi_{S}, \pi_{t}, \pi_{T}$ will be taken assuming all other variables are held constant.

We summarize various conditions on $p$ and $q$ for results to hold. Most of these are cited from [12] and the references therein.

\subsection{Conditions}

C1 $p, q \in C^{0,1}\left(R^{2}\right)$ are globally bounded above.

C2 $q$ is globally bounded from zero across $(s, t)$.

C3 $p$ and $q$ satisfy a global Hölder condition, that is for some parameters $\alpha$ and $K$ :

$$
\left|q\left(s_{1}, t_{1}\right)-q\left(s_{2}, t_{1}\right)\right| \leq K\left[\left|s_{1}-s_{2}\right|^{\alpha}+\left|t_{1}-t_{2}\right|^{\alpha}\right] .
$$

C4 $p, q \in C^{2,1}\left(R^{2}\right)$ and satisfy a global Hölder condi- tion with respect to $S$.

C5 $p, q \in C^{2,1}\left(R^{2}\right)$ and the second derivatives with respect to $s$ are of most polynomial growth.

C6 $p, q$ are locally Lipschitz.

C7 $p, q$ are of at most linear growth.

C8 $q$ is continuous in $t$ and locally Lipschitz in $s$.

C9 $r$ is uniformly bounded and locally Hölder.

\subsection{Kolmogorov Forward Equation}

The probability density $\pi(s, t ; S, T)$ satisfies the Kolmogorov forward Equation (11):

$$
\pi_{T}=-(p \pi)_{S}+\frac{1}{2}(q \pi)_{S S}
$$

This holds under the following conditions [12, Theorem 5.15].

- C1.

- $\mathrm{C} 2$.

- $\mathrm{C} 3$.

\subsection{Kolmogorov Backward Equation}

The density $\pi(s, t ; S, T)$, considered as a function of $s$, also satisfies the Kolmogorov backward equation

$$
\pi_{t}+p \pi_{s}+\frac{1}{2} q \pi_{s s}=0 .
$$

This holds under the following conditions [12, Theorem 5.15]:

- $\mathrm{C} 1$.

- $\mathrm{C} 2$.

\subsection{Feynman-Kac Formula}

Consider the Equation (5), regarded as a parabolic partial differential Equation:

$$
W_{t}+p W_{s}+\frac{1}{2} q W_{s s}-r W=0
$$

where $p, q, r$ are functions of $(s, t)$, as in 5 . This is subject to the boundary condition $W(S, T)=\psi(S)$. In this section, we allow $r$ to be a function of both $(s, t)$.

The solution is given by the Feynman-Kac formula, expressed as a stochastic expectation:

$$
W(s, t)=\mathbf{E}_{P}[\psi(S) v(u, t) \mid s(t)=s]
$$

where $s=s(T)$ and $\psi(S)=W(S, T)$ for a given value of $T$, and

$$
v(t)=\exp -\int_{t}^{T} r(s, \tau) \mathrm{d} \tau .
$$

The function $v(t)$ may be regarded as a generalized discount function for interest.

The Feynman-Kac formula holds under the following conditions:

1) (C5), (C6), (C7) and $\psi \in C^{2}(R)$ and $\psi$ and its derivatives are of at most polynomial growth [12, Theorem 6.2].

2) (C7), (C8), (C9) and $\psi \in C^{0}(R)$ and is of at most polynomial growth [21, Theorem 5.5].

\section{Girsanov's Theorem}

Both the physical process 1 and risk-neutral process 10 for $s$. can be simplified under Itô's lemma by making the transformation $z=\ln s$ Thus in logarithmic terms the physical process can be described by:

$$
\mathrm{d} z=z_{s} \mathrm{~d} s+\frac{1}{2} z_{s s}(\sigma s)^{2} \mathrm{~d} t=\left(r-\frac{1}{2} \sigma^{2}\right) \mathrm{d} t+\sigma \mathrm{d} B
$$

and the physical process by:

$$
\mathrm{d} z=\left(\mu-\frac{1}{2} \sigma^{2}\right) \mathrm{d} t+\sigma \mathrm{d} B .
$$

Where $r, \mu$ and $\sigma$ are non-stochastic, with $r \leq \mu$, this provides explicit solutions for the physical and risk neutral densities $\rho$ and $\bar{\rho}$ in $z$, which obey the forward equations:

$$
\rho_{T}=-\left[\left(r-\frac{1}{2} \sigma^{2}\right) \rho\right]_{Z}+\frac{1}{2}\left(\sigma^{2} \rho\right)_{Z Z}
$$

and

$$
\bar{\rho}_{T}=-\left[\left(r-\frac{1}{2} \sigma^{2}\right) \bar{\rho}\right]_{\mathrm{Z}}+\frac{1}{2}\left(\sigma^{2} \bar{\rho}\right)_{\mathrm{ZZ}} .
$$

For example $\rho(z, t ; Z, T)$ is normal with mean 


$$
\int\left(r-\frac{1}{2} \sigma^{2}\right) \mathrm{d} \tau
$$

have

and variance $\int \sigma^{2} \mathrm{~d} \tau$. Letting $P=\int r \mathrm{~d} \tau$ and

$$
Q=\int \sigma^{2} \mathrm{~d} \tau
$$

we have

$$
\rho(z, t ; Z, T)=\frac{\mathrm{e}^{-} \frac{\left(Z-P+\frac{1}{2} Q\right)^{2}}{2 Q}}{\sqrt{2 \pi Q}}
$$

and thus

$$
(\ln \rho)_{Z}=^{-} \frac{Z-P+\frac{1}{2} Q}{Q}
$$

On the other hand the density of the stock price $S$ is given by

$$
\pi=\frac{\rho}{S}
$$

with $Z=\ln S$, so that

$$
(\ln \pi)_{S}=(\ln \rho)_{S}-\frac{1}{S}=\frac{1}{S}\left(\frac{\rho_{Z}}{\rho}-1\right) .
$$

Therefore to show that $(\ln \pi)_{S} \leq(\ln \bar{\pi})_{S}$, it suffices to show that $(\ln \rho)_{Z} \leq(\ln \bar{\rho})_{Z}$. This can be shown directly from 31. However we take an approach that illustrates a relationship with Girsanov's theorem, and allows a generalization to the case where the parameters are stochastic.

Make the transformation $X=Z+R(T)$. We then

$$
\begin{gathered}
\rho_{T \mid Z}=\rho_{T \mid X}+\rho_{X} R_{T} \\
{\left[\left(r-\frac{1}{2} \sigma^{2}\right) \rho\right]_{Z}=\left[\left(r-\frac{1}{2} \sigma^{2}\right) \rho\right]_{X}} \\
\left(\sigma^{2} \rho\right)_{Z Z}=\left(\sigma^{2} \rho\right)_{X X}
\end{gathered}
$$

We then have from 29:

$$
\rho_{T \mid X}=-\left[\left(r-\frac{1}{2} \sigma^{2}+R_{T}\right) \rho\right]_{X}+\frac{1}{2}\left(\sigma^{2} \rho\right)_{X X}
$$

Let $\mu=r+R_{T}$ (so that the risk premium $\mu-r$ is not stochastic). Then:

$$
\rho_{T \mid X}=-\left[\left(\mu-\frac{1}{2} \sigma^{2}\right) \rho\right]_{X}+\frac{1}{2}\left(\sigma^{2} \rho\right)_{X X}
$$

It may be concluded from comparing this equation with 30 that $\bar{\rho}(x, t ; X, T)=\rho(z, t ; X-R, T)$, so that changing variables:

$$
[\ln \bar{\rho}(Z, T)]_{Z}=[\ln \rho(Z-R, T)]_{Z} .
$$

If $r \leq \mu$, then $R \geq 0$, so that $(\ln \rho)_{Z} \leq(\ln \bar{\rho})_{Z}$ holds if:

$$
[\ln \rho(Z, T)]_{\mathrm{ZZ}} \leq 0 .
$$

This last condition clearly holds in the case of non-stochastic parameters as in 31 . However it is a condition on the risk neutral process only, and may hold in other cases where the stock volatility $\sigma$ is stochastic. 